\title{
Elf: Computer Automation and Error Correction for a Microwave Network Analyzer
}

\author{
WYMAN L. WILLIAMS, STUDENT MEMBER, IEEE, RICHARD C. COMPTON, STUDENT MEMBER, IEEE, AND
} DAVID B. RUTLEDGE, MEMBER, IEEE

\begin{abstract}
A microwave measurement system has been developed which combines a personal computer (PC) and a conventional vector network analyzer to yield a full complex-error-corrected automatic network analyzer. The system consists of a Hewlett-Packard HP 8410C network analyzer, an HP 8350B sweep oscillator, and an IBM PC. A 3000-line computer program, called Elf, runs on the PC performing calibration and measurement algorithms and providing a flexible, menu-oriented user interface. The system, when calibrated, achieves a worst-case measurement error vector of magnitude $\leq \boldsymbol{0 . 0 2}$ for transmission and reflection coefficient measurements over the 2- to 12.4$\mathrm{GHz}$ frequency range and has a measurement speed of three frequency points/s. Elf provides an inexpensive method for upgrading the HP 8410 to achieve the high accuracy of an automatic network analyzer.
\end{abstract}

\section{INTRODUCTION}

A MICROWAVE VECTOR network analyzer measures the complex scattering parameters of a microwave device or circuit. These $S$-parameters, which relate incident and reflected travelling voltage waves at the ports of the device under test, are the set of network parameters most commonly used for the design of linear circuits at microwave frequencies. Fig. 1 shows one configuration in which a vector voltmeter and reflection-transmission test set are combined to yield a network analyzer. Directional couplers in the test set sample the incident and reflected signals at one port, and the transmitted signal is detected at the other test port. A coaxial relay selects which signal is presented to the vector voltmeter for complex ratio measurement. To perform a full $S$-parameter measurement, the device under test must be flipped end for end once during the measurement, to observe the reflection at both its ports, and the transmission through it in both directions.

With this configuration, the quantities the vector voltmeter measurements will be the true $S$-parameters only to the extent that certain rather stringent requirements are met. The two directional couplers must be identical and have very high directivity. The two test ports of the system must present the characteristic impedance desired for the measurement (usually $50 \Omega$ ), and the frequency responses of the signal paths for the incident, reflected, and

Manuscript received May 14, 1987; revised July 27, 1987. This work was supported in part by Caltech's Program in Advanced Technology, the National Science Foundation, and the Fannie and John Hertz Foundation.

The authors are with the Division of Engineering and Applied Science at the California Institute of Technology, Pasadena, CA 91125.

IEEE log number 8717646 .

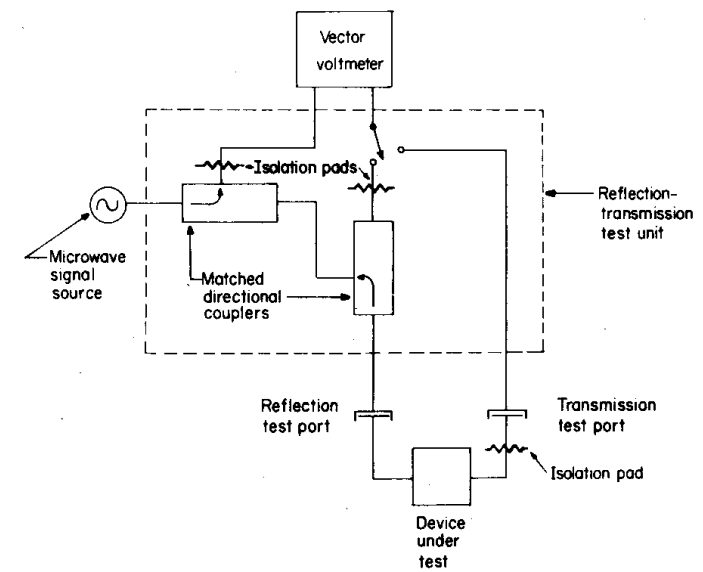

Fig. 1. A vector voltmeter with a reflection-transmission test set can be used to measure the $S$-parameters of an unknown network. The systematic errors of such a network analyzer can be found through calibration, and measurement errors removed in closed form when certain conditions are met.

transmitted signals must matched in amplitude and phase. Meeting all these criteria over a broad microwave frequency band is difficult, but happily with the advent of inexpensive desktop computers, it is no longer necessary; the large errors which can result from imperfections in the reflection-transmission test set are systematic and can be calibrated out by measuring a few known standards.

Use of a computer to correct linear systematic errors in a network analyzer is the basis of such advanced instruments as the Hewlett-Packard HP 8510 network analyzer. Our group at Caltech possesses a less expensive HP 8410C network analyzer, and has a need to make accurate network measurements. It was found that by incorporating an IBM PC with this analyzer, many of the features of the advanced analyzers could be realized at greatly reduced cost. Elf, a 3000-line Pascal program, was written to perform the tasks necessary for calibration, measurement, and display of the data.

\section{THEORY}

It has been previously shown [1]-[3] that for a network analyzer of the type shown in Fig. 1, only three complex constants are required to completely characterize the systematic errors which arise in making reflection measurements at a given frequency. This result is general. That 


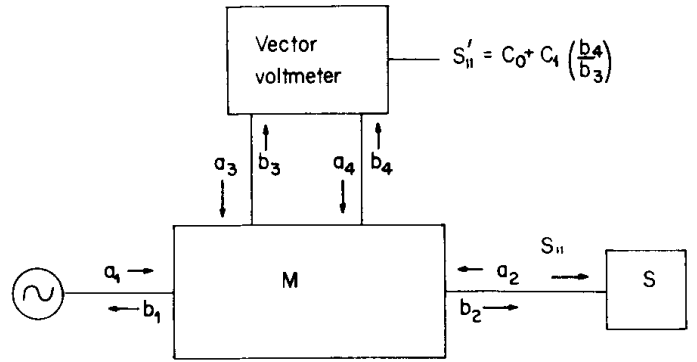

Fig. 2. A reflectometer consisting of an arbitrary linear four-port network and a vector voltmeter. The systematic errors of such an instrument at a given frequency are completely characterized by three complex numbers.

is, it holds for the reflectometer of Fig. 2, where $M$ is an arbitrary linear four-port, and the vector voltmeter has an offset and a frequency response term such that its output reading, $S_{11}^{\prime}$ is given by $S_{11}^{\prime}=C_{0}+C_{1}\left(b_{4} / b_{3}\right)$ where $C_{0}$ and $C_{1}$ are complex constants. For this reflectometer, the relation between the observed reflection coefficient, $S_{11}^{\prime}$, and the actual value $S_{11}$ at a given frequency is given by

$$
S_{11}^{\prime}=\frac{S_{11}+A}{B S_{11}+C} \text {. }
$$

The three complex constants, $A, B$, and $C$, of this bilinear transform can be determined by measuring three known impedance standards. When measuring an unknown, then, the transform can be inverted to yield the actual value of $S_{11}$, given $S_{11}^{\prime}$.

The fact that the two are related by a bilinear transform allows the introduction of a simple error model which is used in many discussions of reflectometers. Making a measurement with a reflectometer of the type shown in Fig. 2 is equivalent to making a measurement with an ideal reflectometer with a linear "error two-port" placed between the reflectometer's test port and the impedance to be measured.

The situation is a bit more complicated when an unknown two-port device is to be measured. Transmission as well as reffection must be measured. The two measurement configurations required are shown in Fig. 3. These correspond to the two switch positions in the measurement system of Fig. 1. The reflection measurement of Fig. 3(a) is the same as above except that here we measure the input reflection coefficient of the two-port with its second port terminated in $\Gamma_{L}$, the impedance of the transmission test port. When performing the transmission measurement, port 2 of the device under test is connected directly to one port of the vector voltmeter, as shown in Fig. 3(b), so the meter measures $b_{5} / b_{3}$.

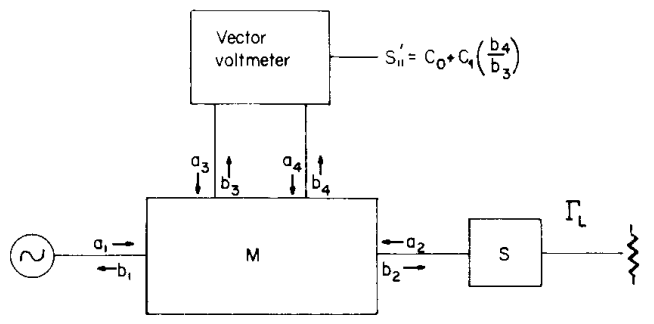

(a)

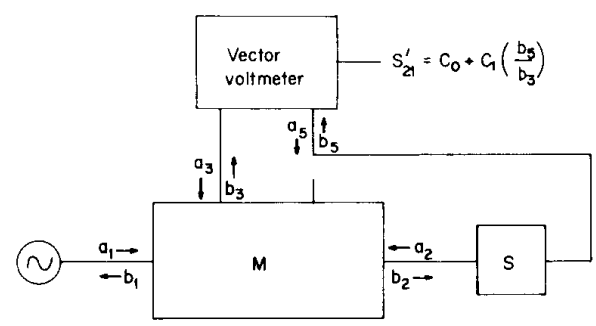

(b)

Fig. 3. The two measurement systems corresponding to the two switch positions in Fig. 1. The systematic errors of this $S$-parameter measuring system are characterized by six complex constants when the criteria outlined in the text are met.

These two measurements give two nonlinear equations in the four $S$-parameters of the unknown device. Flipping the network end for end and repeating these two measurements yields a total of four equations in the four unknown $S$-parameters. These four nonlinear equations cannot, in general, be solved in closed form [2]. The closed-form solution which is commonly known as the 12 term error model for $S$-parameter measurements is only valid if certain conditions are met by the measurement system. These conditions are that the impedance seen by the four-port network $M$ looking out port 4, and the impedance $\Gamma_{L}$ seen by the unknown two-port, do not change when the measurement system is switched from transmission to reflection measurement. If these impedances are switch-dependent, then the equations for the $S$-parameters must be solved iteratively.

To avoid this switch dependence, the hardware of commercial reflection-transmission test sets is engineered to isolate the detector switch from the RF measurement ports of the analyzer. Attenuators are placed between the fourport network $(M)$ and the vector voltmeter, and between port 2 of the device under test and the transmission return port. Also, the input port of the vector voltmeter is matched to the line as well as possible, and switches which terminate unselected lines in $50 \Omega$ are used. Assuming this isolation, the true $S$-parameters can be written in terms of the observed (primed) values as

$$
\begin{aligned}
& S_{11}=\frac{E^{2}\left(A-C S_{11}^{\prime}\right)\left(B S_{22}^{\prime}-1\right)-\Gamma_{L}(A B-C)^{2}\left(S_{12}^{\prime}-D\right)\left(S_{21}^{\prime}-D\right)}{E^{2}\left(B S_{11}^{\prime}-1\right)\left(B S_{22}^{\prime}-1\right)-\Gamma_{L}^{2}(A B-C)^{2}\left(S_{12}^{\prime}-D\right)\left(S_{21}^{\prime}-D\right)} \\
& S_{12}=\frac{E(A B-C)\left(S_{12}^{\prime}-D\right)\left[\left(B S_{11}^{\prime}-1\right)-\Gamma_{L}\left(A-C S_{11}^{\prime}\right)\right]}{E^{2}\left(B S_{11}^{\prime}-1\right)\left(B S_{22}^{\prime}-1\right)-\Gamma_{L}^{2}(A B-C)^{2}\left(S_{12}^{\prime}-D\right)\left(S_{21}^{\prime}-I\right)}
\end{aligned}
$$




$$
\begin{aligned}
S_{21} & =\frac{E(A B-C)\left(S_{21}^{\prime}-D\right)\left[\left(B S_{22}^{\prime}-1\right)-\Gamma_{L}\left(A-C S_{22}^{\prime}\right)\right]}{E^{2}\left(B S_{11}^{\prime}-1\right)\left(B S_{22}^{\prime}-1\right)-\Gamma_{L}^{2}(A B-C)^{2}\left(S_{12}^{\prime}-D\right)\left(S_{21}^{\prime}-D\right)} \\
S_{22} & =\frac{E^{2}\left(A-C S_{22}^{\prime}\right)\left(B S_{11}^{\prime}-1\right)-\Gamma_{L}(A B-C)^{2}\left(S_{12}^{\prime}-D\right)\left(S_{21}^{\prime}-D\right)}{E^{2}\left(B S_{11}^{\prime}-1\right)\left(B S_{22}^{\prime}-1\right)-\Gamma_{L}^{2}(A B-C)^{2}\left(S_{12}^{\prime}-D\right)\left(S_{21}^{\prime}-D\right)} .
\end{aligned}
$$

Thus we see that only six complex constants, $A, B, C, D$, $E$, and $\Gamma_{L}$ are required to model the linear systematic errors of the reflection-transmission measurement.

In addition to the assumption of switch-independence, another assumption must be made to arrive at the result above. It is assumed that the only coupling between the reflection and transmission test ports is through the network under test: there are no RF leakage paths (the "leakage path" which give rise to the $D$ term above is in fact just the offset term in the vector voltmeter's response). The assumption of no RF leakage is good in most systems, since coaxial relays, which have very high isolation, are typically used to switch the signal paths. If significant leakage paths do exist, the expressions above become considerably more complex. More constants and cross terms of the $S$-parameters are involved, and inversion in closed form is impossible.

\section{Hardware Description}

Fig. 4 shows a block diagram of the hardware of the Elf measurement system. The computer communicates with the rest of the system through two interface cards, a general-purpose A/D-and-D./A card [4] and an IEEE-488 bus interface card [5]. In the configuration shown, the IEEE-488 card is used to set the frequency of the sweep oscillator, and the A/D card reads the value of the network analyzer measurement from the horizontal and vertical outputs of the HP 8414A polar display which is used with the analyzer.

The reflection-transmission test unit was a HewlettPackard HP 8743B, which features 7-mm precision connectors and couplers operating from 2 to $12.4 \mathrm{GHz}$. Any reflection-transmission test unit with $7-\mathrm{mm}$ connectors could be substituted for this unit.

When the system was initially constructed, an IEEE488 compatible sweeper was not available, so there is another system configuration option which uses any frequency-proportional-to-voltage microwave source. In this configuration, a voltage from the computer's D/A board is used to set the sweeper frequency, assuming that this frequency is related linearly to voltage. The frequencies at the ends of the sweep are measured by an IEEE-488 compatible microwave counter (HP 5350A) to determine the constants of the linear relation between voltage and frequency.

Fig. 5 shows a photograph of the measurement system. At the top of the instrument rack is the HP $5250 \mathrm{~A}$ microwave counter. Below it is the HP $8410 \mathrm{C}$ with the HP 8414A polar display. Next is the HP 8350B sweeper with HP 83592A RF plug-in, which has a frequency range of 0.01 to $20 \mathrm{GHz}$. At bottom is an HP 8743B reflectiontransmission test set.

\section{Software Description}

Elf is written in Pascal, using the Turbo Pascal [6] compiler. It uses the 8087 numeric coprocessor in the IBM PC to increase calculation speed. As noted above, Elf is a menu-oriented program. Fig. 6 shows the hierarchy of Elf's menus. At start-up, the main menu is displayed. Four options on the main menu select immediate actions to be performed and the remaining four select sub-menus from which additional options can be selected. The first half of the main menu controls the interactions of Elf with the hardware, dealing with mode selection, calibration, and measurement. The second half is concerned with manipulation, storage, and display of the data after measurement. Elf does not have a continuous measurement mode. After each swept-frequency measurement is made, the data are error-corrected and stored, and may then be saved or displayed in a number of formats.

\section{A. Mode Selection, Calibration, and Measurement}

The main menu display shows the following:

\section{MAIN MENU}

1. Select number of frequency points.

2. Select operating mode.

3. Select frequency range.

4. Calibrate analyzer.

5. Make Measurement.

6. Save data to disk.

7. Line stretcher.

8. Print data.

9. Exit.

From this starting point, the system can be calibrated by going through the first four menu items in sequence. Then, measurements may be made using item 5 , and the measured data manipulated and displayed by the remainder of the menu items. Once calibrated, the system may be used for as many measurements over the calibrated range as desired.

First, the number of measurement points is selected. Calibration and measurement will be performed at this number of frequency points evenly spaced between the start and stop frequencies selected below.

Next, the operating mode must be selected. Selecting ' 2 ' will result in a sub-menu which allows the user to select between reflection measurement and full $s$-parameter measurement modes, with the option of sliding load calibration in either mode.

The choice between reflection and full $S$-parameter measurements is offered since a full $S$-parameter calibration and measurement is unnecessarily time consuming 


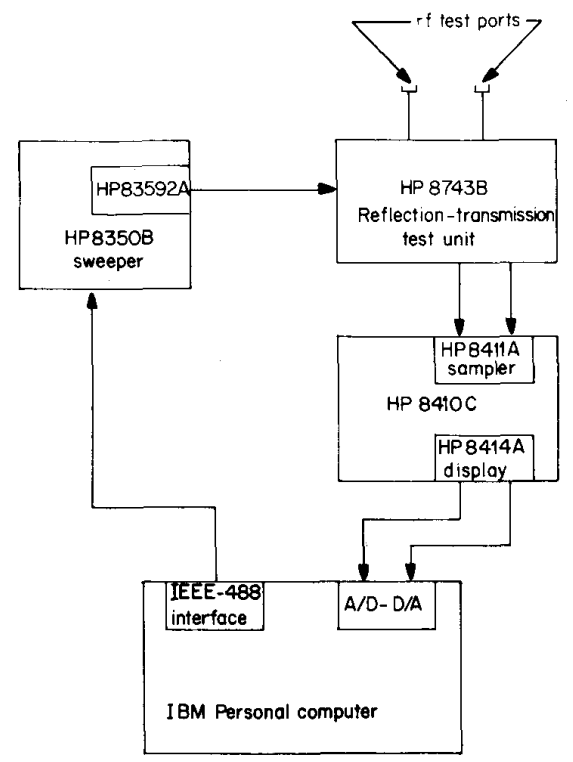

Fig. 4. A block diagram of the Elf system. The computer controls the measurement by setting the sweeper frequency and instructing the user to throw switches on the reflection-transmission test unit.

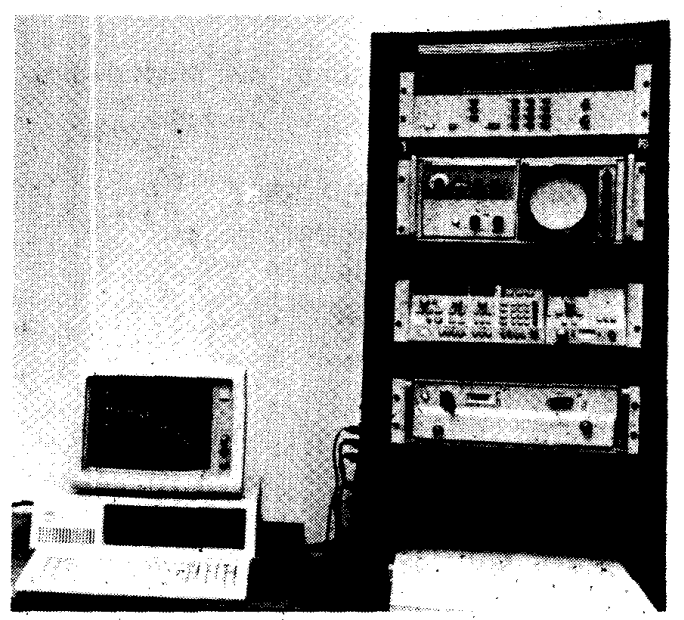

Fig. 5. The Elf system as implemented at Caltech, using an HP $8350 \mathrm{~B}$ sweeper with HP 83592A rf plug-in, an HP $8410 \mathrm{C}$ network analyzer with HP 8414A display unit and HP 8411A harmonic frequency converter, and an HP $8743 \mathrm{~B}$ reflection-transmission test unit. The IBM personal computer is shown with the EGA display.

when one only wishes to measure a reflection coefficient. The sliding load calibration option is offered for better accuracy in high-frequency measurements. Imperfections in the precision $50-\Omega$ load used as one of the calibration standards become more significant with increasing frequency, causing errors in the determination of the zeroreflection point. The locus of reflection coefficients of a sliding load as it moves is a small circle about the zeroreflection point, so the point can be determined even in the presence of imperfections in the load. The effect is to calibrate to the characteristic impedance of the line the load is sliding in, and not to the load itself.

Having selected the measurement mode and returned to

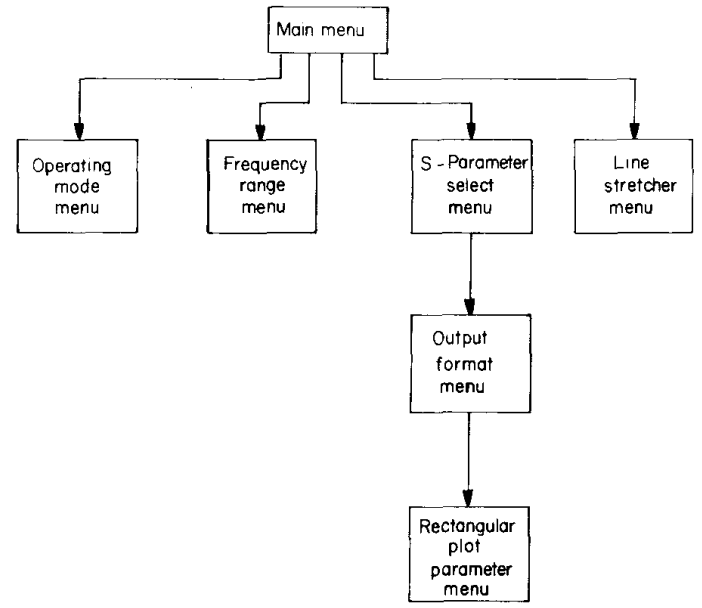

Fig. 6. Elf's menu tree.

the main menu, ' 3 ' is entered to select the frequency range of the measurement. The following sub-menu results:

\section{FREQUENCY RANGE SELECTION MENU \\ Sweeper Frequency Range}

1. HP $8620 \mathrm{C}$ 4. Measure full range

2. HP 694C 5. Select frequency sub-range

3. HP $8350 \mathrm{~B}$

6. Exit

The first two choices were non-IEEE-488 sweepers which were modified to be voltage controlled sources. Choice three is the IEEE-488 controlled sweeper. Choice four is used with the voltage-controlled sweepers: it uses the counter to measure the extremes of the frequency sweep of the source. Selection five can then be used to choose a sub-range of the frequency sweep of the source. When the IEEE-488 controlled sweeper is used, only step five is needed to select the frequency sweep range. Choice six returns to the main menu.

With the operating mode, frequency range, and number of points selected, the analyzer is ready for calibration. The question of choice of calibration standards then arises. Due to small nonlinearities which are not calibrated out by the procedure described in Section II, the network analyzer tends to be most accurate when measuring reflection and transmission coefficients close to those of the calibration standards used. Since the only requirements for calibration is three (or six) known standards, the standards can be chosen to be close to the expected measurement values for maximum accuracy. For example, when measuring a high impedance device at the end of a transmission line, one might choose an open circuit on the same length of line as one of the calibration standards. In Elf, a default general-purpose set of standards is programmed in. To choose another set of standards, a mathematical model of the new standards as a function of frequency 
must be entered. This involves changing a few lines of Elf's Pascal source code, and recompiling it. The default set of standards is a short circuit, an open circuit, a matched load, and a straight through (the matched load and straight though are measured in both reflection and transmission to give the total of six standards). The matched load can be either fixed or sliding, as noted above. The short circuit and open circuit models assume that a Wiltron model 22A50 7-mm precision open/short is used. This standard has a shielded open circuit on one end and a delayed short circuit on the other. The short circuit is 0.050 in from the connector reference plane, and is modeled as this length of transmission line terminated in a perfect short circuit. The shielded open is modeled as a frequency-dependent capacitance. The model used is from [3], $C=0.079+4 \times 10^{-5} f^{2}$ where $C$ is the capacitance in picofarad's, and $f$ is the frequency in gigahertz.

When the calibration option is selected from the main menu, a series of messages appear on the screen guiding the operator through the calibration procedure. At any step the procedure may be aborted, or the previous step may be repeated. Similarly, when option five, 'Make measurement' is selected a series of messages guide the operator through the measurement procedure.

\section{B. Manipulation, Storage and Display}

By stepping through the first five main menu items, the analyzer is calibrated and a measurement performed. The remaining menu items allow the measurement data to be manipulated in a limited way, stored on disk, and displayed in several formats.

Entering menu item seven calls up the software line stretcher. This allows the user to apply a phase shift, linear with frequency, to the measured phase data, thus effectively shifting the position of the reference plane from that at which the analyzer was calibrated. This is often convenient when the device under test is unavoidably separated from the calibrated reference plane by a length of $50-\Omega$ line. From the line stretcher menu, the user can enter a length of line to move the reference plane, or Elf can perform a least squares linear fit of the phase data to arrive at an estimate of the line stretch value.

Item six on the main menu allows the user to store all measured and line-stretched data, as well as all calibration constants, in a disk file. The data are dumped into an ASCII text file, organized in columns with appropriate headings. There is no provision for reading data or calibration constants back in from disk files.

Finally, item eight on the main menu allows the user to display the measured or line-stretched data, either as a table or on a Smith chart or rectangular plot. One quantity may be plotted at a time and the user may choose a magnitude, phase, or decibel plot for the rectangular plot, with any scale desired. There are two versions of Elf. One supports the IBM Color Graphics Adapter (CGA), giving a $640 \times 200$ pixel single color graphics plot. The other supports the IBM Enhanced Graphics Adapter (EGA), giving a $640 \times 350$ pixel two-color plot. In either case, hard

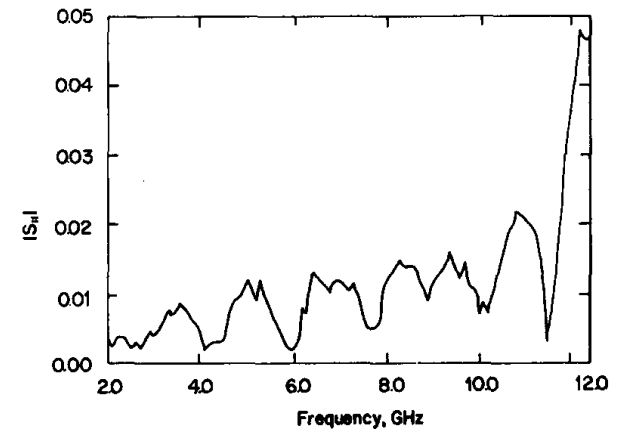

(a)

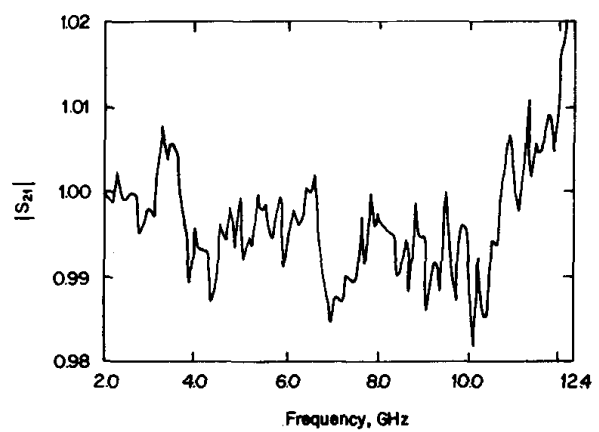

(b)

Fig. 7. (a) Measurement of $S_{11}$ and (b) $S_{21}$ of a length of precision coaxial line. Worst-case reflection or transmission coefficient error is estimated at \pm 0.005 for frequencies from 2 to $5 \mathrm{GHz}, \pm 0.01$ for 5 to $10 \mathrm{GHz}$ and \pm 0.02 for 10 to $12.4 \mathrm{GHz}$. Over this entire range, performance is limited by electronic noise, not repeatability of microwave connections.

copy of the plot can be generated on a dot matrix printer by use of a graphics screen dump.

Elf differs from the system of [3] chiefly in the addition of the transmission measurement capability. Measurement speed and accuracy are about the same. Also, Elf adds the software line stretcher, and rectangular plotting capability. Elf does not, however, provide for easy input of calibration models by the user as does the NRAO system.

\section{Sample Measurement}

Fig. 7 shows magnitude plots of the measured values of $S_{11}$ and $S_{21}$ for a 10.35 -cm length of precision air coaxial line (Hewlett-Packard model 11566A) over the frequency range of 2 to $12.4 \mathrm{GHz}$. The measurements deviate from the ideal values of zero and unity, respectively, by approximately \pm 0.02 in magnitude for the transmission measurement and \pm 0.05 for the reflection measurement. The maximum phase deviation is about 2 degrees. These deviations result from imperfections in the line and its connectors, and from measurement error. Imperfections in the line can be seen in the reflection coefficient data as a series of ripples spaced approximately at multiples of $1.45 \mathrm{GHz}$ which is the half-wave resonant frequency of a $10.35-\mathrm{cm}$ line. Experiments indicate that measurement error is generated mainly by electronic noise and not by repeatability problems with the microwave 
connections: the same level of error occurs when repeatedly measuring the calibration standards without disconnecting and reconnecting them.

The measurement speed of the Elf system is about three frequency points $/ s$ in reflection measurement mode. The sweep speed is about the same in $S$-parameter mode, but here the speed of measurement is limited by the requirement of manually reversing the device under test. We estimate that the magnitude of the error vector of this system is as follows: 0.005 for frequencies from 2 to $5 \mathrm{GHz}$; 0.01 for 5 to $10 \mathrm{GHz} ; 0.02$ for 10 to $12.4 \mathrm{GHz}$. The accuracy and repeatability of the frequency setting for calibration and measurement is determined by the particular sweep oscillator used: for the HP 8350 the same frequency setting command is transmitted to the sweeper over the IEEE-488 bus at matching frequency points for calibration and measurement. For the voltage-controlled oscillators, the same 12 -bit value is sent to the $D / A$ board.

\section{Conclusion}

An automatic microwave network analyzer has been constructed using the HP $8410 \mathrm{C}$ network analyzer, an IBM PC, and an IEEE-488 controlled sweeper. It has been in service for over a year at Caltech, and was used by students in a microwave IC design course for laboratory measurements. It has proven versatile and reliable, with an accuracy of \pm 0.02 in magnitude and 2 degrees in phase for measurement of $S$-parameters over the 2 - to $12.4-\mathrm{GHz}$ frequency range. Elf, a Pascal program which runs on the IBM PC performs the calibration, measurement, and display routines for the network analyzer. Those interested in obtaining copies of this software are invited to contact the authors at Caltech.

\section{ACKNOWLEDGMENT}

The authors thank B. Allen of TRW and S. Weinreb of the National Radio Astronomy Observatory for helpful discussion and suggestions during the development of Elf.

\section{REFERENCES}

[1] J. G. Evans, "Linear two-port characterization independent of measuring set impedance imperfections,"' Proc. IEEE, vol. 56, no. 4, pp. 754-755, Apr. 1968.

[2] A. A. M. Saleh, "Explicit formulas for error correction in microwave measuring sets with switching-dependent port mismatches," IEEE Trans. Instrum. Meas., vol. IM-28, no. 1, Mar. 1979.

[3] Larry R. D'Addario, "Computer-corrected reflectometer using the HP8410 and an Apple II," Nat. Radio Astronomy Observatory Electron. Div. Internal Rep., no. 228, May 1982.

[4] Model DT-2801A from Data Translation, Inc., Marlborough, MA, 01752.

[5] Model PC-488 from Capital Equipment Corp. Burlington, MA 01803.

[6] The Turbo Pascal compiler is a product of Borland International, Scotts Valley, CA 95066.

[7] W. Kruppa and K. F. Sodomsky, "An explicit solution for the scattering parameters of a linear two-port with an imperfect test set," IEEE Trans. Microwave Theory Tech., vol. MTT-19, no. 1, pp. 122-123, Jan. 1971.

[8] S. Rehnmark, "On the calibration process of automatic network analyzer systems," IEEE Trans. Microwave Theory Tech., vol. MTT-22, no. 4, pp. 457-458, Apr. 1974. 